\title{
Sleepless No More: \\ Techniques and Interventions for Sleep Disorders
}

\author{
By Joanna Katsanis* \\ Katharine C. Newman-Smith ${ }^{\dagger}$
}

Disturbed sleep has a significant impact on daytime functioning, mood and quality of life. Sleep disorders are among the most common conditions that affect the general population. Sleep initiation and maintenance difficulties have been associated with mood disturbance, fatigue, occupational impairment, higher morbidity and higher health care costs. Individuals with insomnia often do not seek treatment and when they do they typically initiate over-the-counter sleep medications or alcohol. Furthermore, when they reach out to the medical profession they are, most commonly, prescribed hypnotic medication. There has been increased recognition of the role of psychological and behavioral factors in etiology, assessment and treatment of certain sleep disorders. However, despite this, many mental health professionals are unaware of non-pharmacological treatments for sleep disorders and/or lack the knowledge to recognize and identify sleep problems and implement specific interventions. Sleep disturbance has seldom been the focus of general psychotherapy and empirically validated non pharmacological treatments are rarely used outside sleep disorder centers. The purpose of this paper is to provide mental health professionals with a practical framework for the assessment and management of common sleep disorders seen in clinical practice. Evidence based practice parameters and consensus-based recommendations will be reviewed. Non pharmacological therapies including stimulus control, progressive muscle relaxation, light therapy, sleep restriction, and cognitive-behavior therapy will be presented. Their efficacy when used in clinical practice by non-sleep specialists is well documented. Use of behavioral and psychological treatments for sleep disorders has been found to lead to improvements in sleep quality that are sustained long term and long after treatment completion. Reduction in sleep difficulties improve quality of life and lead to a reduction in hypnotic medication use and dependence.

*Associate Professor, University of Arizona, USA.

${ }^{\dagger}$ Doctoral Candidate, University of Arizona, USA. 


\section{Introduction}

Insomnia refers to sleep disturbance where the individual has problems falling asleep, staying asleep or, upon awakening, has non-restorative sleep. Symptoms of insomnia occur in 33\%-50\% of the adult population. It can be experienced occasionally or persistently. A National Sleep Foundation survey found that occasional insomniacs report on the average 5.2 nights of troubled sleep a month, whereas chronic insomniacs 16.4 (Richardson and Zee, 2006). The most frequent reason for insomnia was work related stress for the occasional insomniacs and family related stress for the chronic insomniacs.

Insomnia can cause daytime impairment or distress. The consequences of insomnia include low energy, poor concentration and memory, fatigue, mood disturbance, psychomotor slowness and overall reduced quality of life. Recent research has even linked insomnia to obesity and depression. Sleep disorders are more likely to affect women, older adults and shift workers. The causes of insomnia can be psychological and/or physiological (Morin et al., 1999).

Stress is one of the most common precipitants of adjustment or transient insomnia. This type of insomnia is quite common in the population and typically resolves when the stressor dissipates. Seventy four percent of people with poor sleep identified stressful life experiences as being associated with the onset of insomnia and sleep problems. These include death of a loved one, break-up of a relationship, interpersonal conflict, and health and occupational stressors. Stress causes emotional, cognitive and physiological arousal that exacerbates sleep difficulties. Bonnet and Arand (1995) found that people with insomnia are more physiologically hyperaroused as indicated by higher whole body metabolic rates, body temperature and heart rate, and central nervous system activation which has been hypothesized to be the underlying cause of insomnia. However, it is not conclusive whether the physiological activation is the result rather than the cause of insomnia (Richardson and Zee, 2006).

Transient insomnia can lead to chronic insomnia if the individual uses maladaptive strategies to cope with his/her lack of sleep. In this case insomnia persists long after the stressor has resolved. In this case poor sleep habits develop when individuals try to cope with the debilitating effects of sleep disturbance. For example, when people have a "difficult" night and do not get an adequate amount of sleep, they may try to compensate by sleeping until the late morning hours or by napping the next day. These approaches can provide temporary relief, but in the long run, disrupt the underlying sleep-wake rhythm. The result is that these compensatory behaviors can make an otherwise temporary sleep disturbance chronic. Similarly, irregular napping during the day is usually followed by a difficulty falling asleep at night, thus perpetuating the irregularity in the sleep-wake rhythm.

Maladaptive sleep habits can be detrimental to sleep. These habits are often referred to as poor sleep hygiene. The most common are irregular sleepawake schedules, irregular napping in the daytime, spending excessive time in bed, or carrying out activities that are not conducive to falling asleep. In addition, an individual may have certain personality traits that heighten his/her 
physiological reaction in the face of stress. For example, one may have the tendency to ruminate over negative events or catastrophize about future events. They may be anxiety prone and engage in unhealthy sleep practices such as lying in bed while awake, or take naps during the day. The above factors place them at an increased risk of developing insomnia in the face of a life stressor (Schutte-Rodin et al., 2008).

The monitoring of the patient's sleep-wake cycle by using daily sleep diaries is important in the assessment of sleep problems and in implementing appropriate changes. In the sleep diaries patients record the number and duration of naps, amount of time they spend in bed and the frequency and duration of night-time awakenings. Sleep diaries do not only help the clinician determine where intervention is needed, but also note when progress is made following certain interventions. Also, in the cases when interventions fail, examination of the sleep diaries can help identify the possible causes for the failure. Individuals may partially comply with the instructions given to them. Partial compliance can adversely affect the sleep-wake cycle and perpetuate sleep problems. Another use of the sleep diaries is that they provide an objective account of the patient's sleep difficulties; the therapist and patient can collaboratively assess the self-reported sleep habits and count the amount of total sleep time across the time period reported. Patients with insomnia often underestimate the amount that they sleep and do not realize that their sleep problems are not as severe as they originally believed. Sleep diaries allow for a reassessment and discussion of the patient's current sleeping times and amounts and thus provide a more objective account of amount of sleep than retrospective reporting.

As indicated thus far, psychological and behavioral factors have been found to contribute to the onset and maintenance of insomnia. In order to address sleep difficulties patients frequently resort to alcohol and/or to overthe-counter sleep aids. When they seek help from their medical health provider pharmacotherapy is most commonly introduced. However, long term use of hypnotic medication has not been found to be beneficial and in fact is contraindicated due to the potential risk for tolerance and dependency.

There are several non-pharmacological interventions that have been developed for the treatment of insomnia with significant success. However, although professionals have been encouraged over the years to integrate these in the pharmacological treatment of insomnia, these methods are largely underused. The purpose of this paper is to introduce clinicians to available non pharmacological treatment methods. Based on extensive research and assessment of available research, the following treatments are considered the gold standard of insomnia treatment.

Initial treatment of insomnia should address any medical condition, psychiatric condition, or substance abuse that precipitated the onset of insomnia. This treatment should involve sleep hygiene instruction and stimulus control procedures. If at follow-up sleep complaints persist introduction of cognitive behavior therapy, relaxation, and sleep restriction should be 
introduced (Morgenthaler et al., 2006). These procedures will be presented below.

\section{Consensus Based Guidelines}

\section{Sleep Hygiene}

Sleep hygiene instructions, patterns of behavior and environments conducive to the promotion of sleep, have been found particularly effective in decreasing the duration and shortening the course of insomnia. Sleep Hygiene Instructions list a series of recommendations that should be introduced to the patient and encourage him/her to follow.

Sleep Hygiene Instructions

Maintain a regular sleep-wake schedule

Keep sleep environment comfortable (pleasant temperature, no light/noise, reduce interruptions e.g. by removing pets)

Avoid:

○ Alcohol, smoking or intense exercise before bedtime

- Caffeine or other stimulants after lunch

- Eating a large meal before going to sleep

- Taking your problems to bed (i.e. ruminate) or stay awake while in bed

- Long naps (> $30 \mathrm{~min}$ ) and not late in the day

\section{Stimulus Control}

Stimulus control therapy (SCT), a standard treatment recommendation by the American Academy of Sleep Medicine (AASM), was the first nonpharmacological intervention developed for chronic insomnia (Bootzin, 1972; Bootzin, 1977; Bootzin and Epstein, 2011; Schutte-Rodin et. al, 2008). SCT has demonstrated the most efficacy as a single-component treatment and is an integral element of many cognitive and behavioral therapies for insomnia such as CBT-I (Morin et al. 1999, 2006). This treatment emphasizes that the inability to initiate sleep is fueled by maladaptive associations formed in the bedroom. Based on operant conditioning principles, the bed and bedroom become negatively associated with behaviors incompatible with sleep such as eating, watching TV, reading, listening to music, playing on the computer, etc. Additional negative associations are formed with feelings of frustration created by an inability to initiate sustained sleep and worry or anxiety about possible next-day symptoms, including daytime sleepiness or a failure to function as needed (Bootzin and Epstein, 2011). These maladaptive behavioral and psychological associations become perpetuating factors of insomnia. The goal of stimulus control is to re-associate the bed and bedroom with sleepcompatible behaviors, and furthermore to reestablish an appropriate sleep-wake schedule. With few instructions, mental health professionals can easily aid their patients in forming new, improved associations of the bed and bedroom to sleep-compatible behaviors improving insomnia symptoms. 


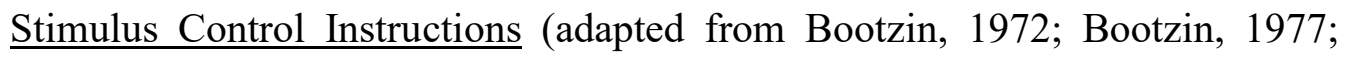
Schutte-Rodin et al., 2008):

1. One should go to bed only when sleepy

2. All other activities should be done elsewhere. That is, reading, watching TV, listening to music, eating, playing on the computer, worrying, etc., should all be done in another room. The only exception is sexual activity, after which one should leave the bed unless sleepy and intending to attempt to fall asleep.

3. If one is unable to fall asleep after about 20 minutes, one should get out of bed and do a different, non-arousing activity in low light - such as light reading, relaxation exercises, or listening to music. It is important not to watch the clock while attempting to initiate sleep, rather estimate the 20 minutes.

4. If one is unable to sleep after returning to bed, repeat step 3 as often as needed during the night.

5. Create a consistent sleep schedule. It is critical always to arise from bed at the same time, even if sleep has been minimal the night before. Take special care to remain consistent regardless of the day of the week (i.e. weekdays or weekends).

6. Napping is prohibited.

When administering this insomnia treatment, it is important for mental health professionals to remind their patients that they may initially feel slightly sleep-deprived due to the number of times they will get out of bed and the lack of napping. Professionals can explain to their patients that this will improve over treatment and in the short-term, be capitalized upon to aid in reassociating the bed with sleep. Over extended wakefulness, the need for sleep - the homeostatic sleep drive-builds. Once sleep is attained, this drive dissipates only to repeat this same process across the next wakeful period (Borbely and Acherman, 1992). Additionally, regularization of the sleep-wake schedule will affect the body's internal circadian clock resulting in a more habitual feeling of sleepiness at the same time every day. This capitalization on the basic sleep physiological processes, behavioral modifications to the bedroom, and a consistent sleep/wake schedule are critical to this treatments success of reducing insomnia severity.

\section{Cognitive Behavior Therapy}

Cognitive Behavior Therapy for Insomnia (CBT-I), a first rate treatment combining the discussed stimulus control and sleep restriction treatments with cognitive therapy aimed at improving cognitive associations with sleep, has been deemed more effective than placebo (Schutte-Rodin et al., 2008) and pharmacological interventions (Mitchell et al., 2012). One of the main components is providing psychoeducation about sleep and sleep problems, addressing the maladaptive patterns people have developed in an attempt to deal with their sleep. Psychoeducation attempts to normalize the sleep-wake 
cycle by encouraging sleep hygiene practices such as regular use of naps during the day, the maintenance of regular sleep-wake schedule and the avoidance of caffeine, alcohol or nicotine close to bedtime. In addition, individuals are discouraged from exercising, or engaging in anxiety inducing activities (e.g., worrying) before bedtime. Patients are encouraged to examine their sleep environment and determine possible factors that interfere with their sleep e.g., if their mattress is uncomfortable, to purchase a mattress that suits their needs; if their sleep environment is too bright, to purchase a sleep mask or if the environment is too noisy to use white noise to eliminate background traffic noise. While white noise generators can be used for this purpose an inexpensive way to simulate a similar effect is to set the radio dial at an unused frequency. Increase in daytime physical activity also helps with sleep (Bootzin and Epstein, 2011).

Often patients with insomnia endorse anxious and dysphoric cognitions (Schutte-Rodin et al., 2008). These beliefs correlate with sleep disturbance and emotional distress. CBT-I focuses on altering maladaptive cognitions and replacing them with adaptive and less stress invoking beliefs. For example, people with insomnia often overestimate the impact of their sleep difficulties in their daily lives. They tend to worry over their loss of ability to fall asleep and believe that lack of sleep will have detrimental effects on both their physical as well as mental health. A viscous cycle often develops where their catastrophizing leads to greater emotional arousal which impedes their ability to fall asleep and which in turn results in to the intensification of their fears. The first step in addressing this problem is to identify the presence and nature of maladaptive beliefs through inquiry or use of standardized measures (e.g. Dysfunctional Beliefs and Attitudes about Sleep Questionnaire; Morin, Vallieres and Ivers, 2007). The clinician can then provide psychoeducation regarding the impact of dysfunctional sleep cognitions, dispel any misconceptions the patient may hold about the consequences of insomnia and alter unrealistic sleep expectations.

\section{Sleep Restriction}

Sleep restriction therapy identifies excessive time in bed as a perpetuating factor of chronic insomnia (Spielman et al., 1987a). Despite actual time asleep being minimal, insomniacs seem to prolong their time in bed, possibly in hopes of increasing the opportunity they might sleep (Speilman, et al., 1987a; Schutte-Rodin et al., 2008). Similar to stimulus control, the inability to sleep may co-occur with negative sleep associations, including frustration and anxiety or worry due to lack of sleep. Also a recommended treatment by AASM, the goal of sleep restriction is to improve sleep efficiency by restricting the amount of time in bed to only the amount of time spent asleep as well as creating a consistent sleep/wake schedule. Sleep restriction also results in mild sleep deprivation because the patient's time in bed is curtailed to a shortened period. Similar to the principles of stimulus control, this mild sleepdeprivation capitalizes on the increase in the homeostatic sleep drive, increasing the likelihood of falling asleep at the designated time. 
Sleep Restriction Instructions (adapted from Spielman et al., 1987b; SchutteRodin et al., 2008)

1. A two-week sleep diary is needed to establish baseline sleep habits, this includes: bedtime, wake time, total time in bed, time to fall asleep (sleep latency), arousals (number of times the patient wakes in the night), and wake after sleep onset (amount of time awake during arousals). Using these measures, the sleep efficiency is calculated: (Estimated Total Sleep Time/Time in Bed) $x 100 \%$. Sleep diaries should be utilized daily throughout the entirety of treatment.

2. Time in bed is restricted to the typical time asleep, i.e. if the patient is in bed for 10 hours, but sleeping 5 hours, the time in bed is restricted to 5 hours. Time in bed should never be less than 4.5 hours.

3. A sleep schedule should be created with consistent wake and sleep times working backward from the patient's desired wake time.

4. During treatment, the patient's sleep efficiency should be calculated over 5 days. If sleep efficiency is $85 \%$ or greater, time in bed should be increased by 15 to 30 -minute increments until adequate sleep time is attained (approximately 6-8 weeks).

5. Napping is prohibited

For mental health professionals, administering a sleep restriction treatment to a patient can be difficult. Patients frequently describe difficulty adhering to the prescribed guidelines. Thus if administered, it will be important to educate the patient of this treatment's effectiveness due to the consolidation of sleep into a single period concatenated with a reduction of the total time in bed. This combination results in an improvement in the quality of sleep, wherein the patient will be able to fall asleep more quickly at the designated time.

\section{Relaxation}

Interventions that increase relaxation are useful in the treatment of insomnia, since some insomnia patients are found to be hyperaroused during the daytime as well as at night. Progressive muscle relaxation is most widely used. This procedure involves the tensing and relaxing of the body's major muscle groups. While patients engage in the relaxing of their muscles they are instructed to attend to the changing sensations of tension and relaxation. With practice patients are able to reduce overall physiological arousal as well as presleep arousal and thus improve sleep quality (Morgenthaler et al., 2006).

Progressive muscle relaxation has been found effective in the treatment of insomnia. However, it is worth pointing out that not all patients with insomnia suffer from physiological arousal, thus relaxation procedures should be used with those insomnia patients that present with significant anxiety . 


\section{Internet-based Treatments}

As we continue to transform to an increasingly tech-savvy society, many individuals are turning to internet-based resources for the delivery of healthcare. While mental health professionals may prefer to administer insomnia treatment in-person, the feasibility of delivery may be difficult due to a variety of hurdles, including the patient's preference of treatment modality, the inability of the patient to commit to in-person sessions, and access to healthcare. At present, promising new research suggests that internet-based delivery of Cognitive-Behavioral Therapy for Insomnia (CBT-I) can be a valid, efficacious treatment (i.e. Espie et al., 2012; Ritterband et al., 2009). Applying empirically validated CBT-I, the principles taught are the same as those described above: including rationale of treatment, stimulus control, sleep restriction, cognitive therapy for maladaptive sleep beliefs, etc. Additional important features of web-based delivery include the continued assessment of patient participation based upon the patients completion of assessment based questionnaires and sleep diaries. These improve the fidelity of the therapy administration, forcing the patients to move at an appropriate pace. In some web-based treatments, patients are unable to move to the next module until the appropriate time and completion of diaries has passed (Ritterband et al., 2009), while others use an automated media-rich web application that delivers CBT-I via a 'virtual therapist' (Espie et al., 2012). Empirically based studies of internet-based CBT-I suggest that it is more effective than wait-list control conditions and treatment as usual (Espie et al., 2012; Ritterband et al., 2009). Critically, prior to starting therapy, all participants in both studies were assessed as having met inclusion/exclusion criteria, including a lack of comorbid physical or mental disorders. As such, it will be important for professionals to identify those individuals for whom reputable, empirically based treatments are an appropriate avenue for his/her healthcare delivery.

\section{Circadian Rhythm Disorder Induced Insomnia}

In some situations insomnia is the result of circadian rhythm abnormalities, a dysregulation of the typical sleep-wake cycles. A way to determine this is to ask the patient whether they tend to sleep well but at the "wrong times". For example, people will complain that they are able to sleep well but only if they stay up late or the opposite, that they sleep well if they go to bed very early. Two therapeutic strategies have been found to address this type of insomnia successfully, phototherapy and chronotherapy.

In phototherapy the patient is asked to expose themselves to bright light either when awakening (in cases where their sleep onset occurs later than the desired time) or late in the evening (in cases where their sleep onset occurs earlier than the desired time; Dobson and Zee, 2010). The light initiates typical biological processes, modifying the patient's circadian rhythm to instigate the shift of sleep to an earlier or later time. 
In chronotherapy, a resetting of the typical sleep-wake timing, patients are asked to consistently delay their bedtime by 2-3 hours a day until they reach the desired time. This occurs typically within 10 days. Because patients find it challenging to adhere to this task therefore, for this method to be successful monitoring closely their sleep schedules is imperative (Czeisler et al., 1981).

\section{Differential Diagnosis}

If sleep problems are persistent, the clinician must have a high suspicion for mood and anxiety disorders. Sleep is often altered in depression, and other symptoms include depressed mood, loss of interest or pleasure, changes in appetite, as well as decreased energy, concentration, and motivation. Suicidal thoughts are also possible. Symptoms of anxiety include excessive worry, panic attacks, avoidant behavior, or somatic symptoms. Individuals are often hesitant to discuss symptoms of depression or anxiety with health care providers, as the patients themselves may not understand what they are experiencing and may be struggling with the stigma posed by mental illness in our society. Treatment of underlying psychiatric disorders will likely lead to improvements in sleep, as well as other benefits. Psychotherapy may be adequate and optimal treatment for mild to moderate depression and anxiety, but many patients may require medication for depression or anxiety. Selective Serotonin Reuptake Inhibitors antidepressants are first line treatments for depression and anxiety.

\section{Conclusions}

We hope the present paper highlights the numerous non-pharmacological treatments that mental health professionals can employ to treat their patients' insomnia symptoms. The difficulty of initiating and maintaining sleep over an extended period of time has devastating consequences: impacting cognition, mental health, and physical well-being. Influenced by underlying predisposing conditions, precipitating events, and perpetuating factors, the cognitive and behavioral treatments outlined above utilize basic concepts that can be applied by mental health professionals with any patient experiencing sleep difficulties. Importantly, re-associating the bed and bedroom to sleep compatible behaviors, minimizing sleep-incompatible behaviors, creating a structured sleep and wake schedule, and changing maladaptive thought patterns are critical to improving insomnia symptoms. Each of the treatments discussed has been shown to be effective, resulting in lasting improvements in daytime functioning, decreased sleepiness, and quality of sleep. Importantly, there are multiple modalities with which patients may be treated, of which promising avenues include web-based administration. Lastly, for mental health professionals with minimal experience treating sleep disorders, there are numerous resources and academies focused on the treatment of sleep disorders. 
Take home points:

1. When insomnia appears to be the primary complaint, nonmedication options need to be considered.

2. Clinicians need to teach patients sleep hygiene and other behavioral techniques.

3. Patients should be encouraged to create a consistent sleep/wake schedule.

4. Aid patients in creating positive, sleep-compatible associations in the bedroom. The bed should only be used for sleep and sex.

5. Improve the patient's insomnia coping skills to improve their sleep quality, including creating an appropriate sleep environment, understanding the principles of sleep hygiene, and relaxation strategies.

6. Have the patient get out of bed if sleep is not attained within twenty minutes of attempted initiation.

7. Administer cognitive therapy to aid the patient in restructuring maladaptive sleep beliefs that can instigate anxiety or frustration.

\section{References}

Borbély, A. A., \& Achermann, P. 1992. Concepts and models of sleep regulation: an overview. Journal of Sleep Research, 1, 2, 63-79.

Bonnet, M.H. \& Arand, D.L. 1995. We are chronically sleep deprived. Sleep, 18, 10, 908-911.

Bootzin R.R. 1972. Stimulus control treatment for insomnia. Procceedings 80th Annual Convention American Psychological Association, pp. 395-96. Honolulu, Hawaii.

Bootzin R.R. 1977. Effects of self-control procedures for insomnia. In Behavioral Self-Management: Strategies and Outcomes, ed. R Stuart, pp. 176-96. New York: Brunner/Mazel.

Bootzin, R. R., \& Epstein, D. R. 2011. Understanding and treating insomnia. Annual Review of Clinical Psychology, 7, 435-458.

Czeisler, C.A., Richardson, G.S., Coleman, R.M., Zimmerman, J.C., Moore-Ede, M.C., Dement, W.C., and Weitzman, E.D., 1981. Chronotherapy: resetting the circadian clocks of patients with delayed sleep phase insomnia. Sleep, 4, 1, 1-21.

Dobson, E.R. \& Zee, P.C. 2010. Therapeutics for circadian rhythm sleep disorders. Sleep Medicine Clinics, 5, 4, 701-715.

Espie, C.A., Kyle, S.D., Williams, C., Ong, J.C., Douglas, N. J., Hames, P., \& Brown, J.S. 2012. A randomized, placebo-controlled trial of online cognitive behavioral therapy for chronic insomnia disorder delivered via an automated media-rich web application. Sleep, 35, 6, 769-781.

Mitchell, M. D., Gehrman, P., Perlis, M., \& Umscheid, C. A. (2012). Comparative effectiveness of cognitive behavioral therapy for insomnia: a systematic review. BMC family practice, 13(1), 40.

Morgenthaler, T., Kramer, M., Alessi, C., Friedman, L., Boehlecke, B., Brown, T., Coleman, J., Kapur, V., Lee-Chiong, T., Owens, J., Pancer, J., \& Swick, T. 2006. Practice Parameters for the Psychological and behavioral treatment of insomnia: 
An update. An American Academy of Sleep Medicine Report. Sleep, 29, 11, 1415-1419.

Morin, C.M., Bootzin R.R., Buysse, D.J., Edinger, J.D., Espie C.A., \& Lichstein, K.L. 2006. Psychological and behavioral treatment of insomnia: update of the recent evidence (1998-2004). Sleep, 29, 1398-1314.

Morin C.M., Hauri, P.J., Espie, C.A., Spielman, A.J., Buysse, D.J., \& Bootzin, R.R. 1999. Nonpharmacologic treatment of chronic insomnia: an American Academy of Sleep Medicine review. Sleep, 22, 1134-1156.

Morin, C.M., Vallieres, A., \& Ivers, H., 2007. Dysfunctional Beliefs and Attitudes about Sleep (DBAS): Validation of a Brief Version, Sleep, 30, 11, 1547-1554.

Richardson, G, \& Zee, P.C. 2006. Insomnia: Primary Care Edition. Clinical Symposia, $56,1,1-44$.

Ritterband, L.M., Thorndike, F.P., Gonder-Frederick, L.A., Magee, J.C., Bailey, E.T., Saylor, D.K., \& Morin, C.M. 2009. Efficacy of an Internet-based behavioral intervention for adults with insomnia. Archives of General Psychiatry, 66, 7, 692698.

Schutte-Rodin, S., Broch, L., Buysse, D., Dorsey, C., \& Sateia, M. 2008. Clinical guideline for the evaluation and management of chronic insomnia in adults. Journal of clinical sleep medicine: JCSM: official publication of the American Academy of Sleep Medicine, 4, 5, 487-504.

Spielman, A.J., Saskin, P., \& Thorpy, M.J. 1987a. Treatment of chronic insomnia by restriction of time in bed. Sleep: Journal of Sleep Research \& Sleep Medicine, 10, $1,45-56$.

Spielman, A.J., Caruso, L.S., \& Glovinsky, P.B. 1987b. A behavioral perspective on insomnia treatment. Psychiatric Clinics of North America, 10, 4, 541-553. 
